\title{
High prevalence of dementia among older adults from poor socioeconomic backgrounds in São Paulo, Brazil
}

\author{
Marcia Scazufca, ${ }^{1}$ Paulo R. Menezes, ${ }^{2}$ Homero P. Vallada, ${ }^{3}$ \\ André L. Crepaldi, ${ }^{1}$ Maria Pastor-Valero, ${ }^{4}$ Letícia M. S. \\ Coutinho, ${ }^{2}$ Vanessa D. Di Rienzo ${ }^{1}$ and Osvaldo P. Almeida ${ }^{5}$ \\ ${ }^{1}$ Department of Psychiatry, University of São Paulo Medical School, and Section of Epidemiology, University \\ Hospital, University of São Paulo, Brazil \\ ${ }^{2}$ Department of Preventive Medicine, University of São Paulo Medical School, and Section of Epidemiology, \\ University Hospital, University of São Paulo, Brazil \\ ${ }^{3}$ Department of Psychiatry, University of São Paulo Medical School, Brazil \\ ${ }^{4}$ London School of Hygiene and Tropical Medicine, Department of Epidemiology and Population Health, \\ Distance Learning Program Unit, London, U.K. \\ ${ }^{5}$ Western Australian Centre for Health and Ageing, University of Western Australia and Royal Perth Hospital, \\ Perth, Australia
}

Background: The aim of this study was to determine the prevalence of dementia in a socioeconomically disadvantaged population of older adults living in the city of São Paulo, Brazil.

Methods: A cross-sectional one-phase population-based study was carried out among all residents aged $\geq 65$ in defined census sectors of an economically disadvantaged area of São Paulo. Identification of cases of dementia followed the protocol developed by the 10/66 Dementia Research Group.

Results: Of 2072 individuals in the study, 105 met the criteria for a diagnosis of dementia, yielding a prevalence of $5.1 \%$. Prevalence increased with age for both men and women after age 75 years, but was stable from 65 to 74 years. Low education and income were associated with increased risk of dementia.

Conclusions: The prevalence of dementia among older adults from low socioeconomic backgrounds is high. This may be partly due to adverse socioeconomic conditions and consequent failure to compress morbidity into the latter stages of life. The increasing survival of poorer older adults with dementia living in developing countries may lead to a rapid increase in the prevalence of dementia worldwide. 
Key words: epidemiology, dementia, cognitive impairment, prevalence, aging, developing countries

\section{Introduction}

The prevalence of dementia is increasing worldwide as the population ages (Cohen, 2003). Current estimates indicate that there are 24 million people with dementia worldwide, with approximately $60 \%$ of them living in developing countries (Ferri et al., 2005). The proportion of people with dementia is expected to double every 20 years, and by $204070 \%$ of them will be living in developing countries such as Nigeria, India and Brazil.

Presently available epidemiological studies suggest that the prevalence of dementia is higher in developed than developing-countries (Jorm et al., 1987; Hofman et al., 1991; Hendrie et al., 1995; Breitner et al., 1999; Lobo et al., 2000; 10/66 Dementia Research Group, 2000a; Vas, 2001; de Silva, 2003; Shaji, 2005; Zhang, 2005). However, the number of well-designed surveys aimed at ascertaining the prevalence of dementia in non-industrialized countries is small (10/66 Dementia Research Group, 2000b; Ferri et al., 2005) and fails to express adequately the vast demographic, cultural and socioeconomic heterogeneity of these populations.

During the past few decades a large proportion of older adults living in Latin America moved from rural areas to large urban centres, where they often live in underprivileged areas of the city (Dufour and Piperata, 2004). These people have poor literacy and general health, including high prevalence of cardiovascular diseases (Marins et al., 2007). Low educational attainment and cardiovascular diseases are associated with increased risk of dementia (Newman et al., 2005; Valenzuela and Sachdev, 2006), which suggests that older people living in developing countries have a particularly high risk of developing dementia. These people also lack access to the factors that ultimately lead to successful compression of morbidity (Fries, 1980), and this may lead to earlier onset of illness and greater comorbidity among those living in disadvantaged communities.

Brazil has one of the fastest growing populations of older people in the world (United Nations Department of Economic and Social Affairs, 2002). Those aged 60 years or over currently represent $9 \%$ of the total Brazilian population (Instituto Brasileiro de Geografia e Estatística, 2006), but will make up $24 \%$ the total population by 2050 (United Nations Department of Economic and Social Affairs, 2002). Somewhat surprisingly, there is only sparse systematic information about the prevalence of dementia in Brazil. One study investigated the prevalence of dementia in a medium-size community in the southeast of Brazil (Herrera et al., 2002). In that study, the total prevalence of dementia among subjects aged 65 years or over was $7.1 \%$. There is currently no information about the prevalence of dementia in the socioeconomic disadvantaged large urban centres of Brazil. This study aimed to determine the prevalence of dementia among older adults of low socioeconomic status living in a well-defined region of the city of São Paulo, the largest metropolis in South America. 


\section{Methods}

\section{Study design}

A cross-sectional one-phase population-based study was carried out with all residents aged 65 years or older living in an economically deprived area of São Paulo, Brazil. The present study is part of a collaborative program developed by the 10/66 Dementia Research Group, an international network of investigators, mostly from developing countries (10/66 Dementia Research Group, 2000a; Scazufca et al., 2002).

\section{Study setting and sample}

The city of São Paulo has an estimated population of 10.4 million inhabitants (Instituto Brasileiro de Geografia e Estatística, 2006) and is divided into 31 administrative boroughs, with territorial and population demarcations. The present investigation was carried out in the borough of Butantã, located on the west side of the city. In 2000 the area had 377,576 residents, of whom $6.2 \%$ were aged 65 years or over and $13 \%$ lived in shanty towns. The study was carried out in 66 census sectors (the smallest administrative areas) covering a population of approximately 63,000 residents, representing $17 \%$ of the total population of the Butantã borough. The selection of areas was based on the presence of shanty towns and/or Family Health Program teams (such teams are normally placed in the most disadvantaged areas of the city of São Paulo), but was not limited by them (i.e. they included the entire census areas, not only shanty towns). These areas had the lowest Human Development Indexes of the borough (Secretaria do Governo da Prefeitura de São Paulo, 2004).

Eligible participants were people aged 65 years and over who were residents in the defined census sectors. They were identified and recruited through door knocking of all households within the census sectors boundaries. All eligible subjects were invited to participate, regardless of whether or not other older adults living in the same residence had already consented to take part in the study. Institutionalized individuals were not included.

The sample size was based on the precision of the prevalence estimate, and was calculated to be 2000 subjects, for a prevalence of dementia of $5.0 \%$ with a $95 \%$ confidence interval of $4 \%$ to $6 \%$.

\section{Measurements}

The assessment of dementia was carried out with a harmonized one-phase dementia diagnostic procedure developed by the 10/66 Dementia Research Group for use in population-based studies in developing countries (Prince et al., 2003), and a detailed assessment of the onset and course of the dementia syndrome. This procedure, which was devised to avoid educational and cultural bias in the assessment of dementia, included the Community Screening Instrument for Dementia (CSI-D) (Hall et al., 2000), an adapted version of the CERAD ten-word list learning task with delayed recall, the animal naming verbal fluency task from the CERAD (Welsh et al., 1994), the Geriatric Mental State (GMS; a semi-structured clinical interview for the assessment of mental 
status) (Copeland et al., 1986; Prince et al., 2004), and a structured neurological assessment to ascertain the presence of lateralizing signs, parkinsonism, ataxia, apraxia and primitive "release" reflexes. The interview with the informants consisted of an enquiry about the participant's cognitive and daily function according to the CSI-D (Hall et al., 2000) and a brief history of the participant's functional and cognitive decline based on the History and Aetiology Schedule Dementia Diagnosis and Subtype (HAS-DDS) schedule (Dewey and Copeland, 2001), which was completed when informants reported the presence of decline in the CSI-D. The diagnosis of dementia followed DSM-IV criteria (American Psychiatric Association, 1994) and used all the information collected from the subject and his/her informant. The HAS-DDS also provided information that allowed for the classification of dementia subtypes (American Psychiatric Association, 1994) as Alzheimer disease (AD), vascular dementia (VaD) or mixed dementia. The latter included cases with characteristics of both $\mathrm{AD}$ and $\mathrm{VaD}$, and cases with a history of parkinsonism.

We ascertained age by asking older adults and their informants about the dates of birth of participants, and by collecting the information from participants' identity cards (which are mandatory in Brazil). In the rare instances where there was inconsistency between these sources, the interviewer asked further questions to determine the likely age of the participant.

\section{Procedures}

Eight mental health workers were trained in the use of the research protocol. The assessments were conducted between May 2003 and April 2005. All eligible residents who consented were interviewed. For each participant, an informant was also identified. Informants were co-residents aged 16 years or over, or a relative or friend who was familiar with the participant's life history. Interviews with participants and informants were scheduled to take place at the participants' homes approximately one week after recruitment and, whenever possible, were carried out simultaneously by two different interviewers. These assessments took approximately 90 minutes. For participants with severe mental or physical disabilities, informants were also asked about the participant's socioeconomic characteristics.

\section{Ethical considerations}

Participants provided written informed consent. Those deemed unable to consent because of mental or physical incapacities were recruited on the basis of informants' signed agreement. When participants were illiterate, the information sheet and consent form were read aloud, and verbal witnessed consent was obtained. The study received ethics approval from the Brazilian National Committee for Ethics and Research (CONEP-Brazil).

\section{Statistical analysis}

Data entry was carried out twice, and consistency checks performed. Data analysis was carried out with the software STATA 9.0 (StataCorp, 2005). Age was grouped in five-year age bands from 65 to 84 years, and one group aged 
85 years or older. Monthly income was grouped into five categories (in US dollars equivalent): zero to 85,86 to 127,128 to 246,247 to 500 , and 501 or more. Educational attainment was grouped into three categories: no formal education, incomplete basic education ( 1 to 3 years), and complete basic primary school education (four or more years of school education). The total prevalence of dementia was estimated with its respective $95 \%$ confidence interval $(95 \%$ CI). The prevalence of dementia according to gender and age group was also estimated. We used the direct method to calculate the standardized estimate of the prevalence of dementia, with corresponding $95 \%$ confidence interval (United Nations Department of Economic and Social Affairs, 2005). Briefly, this approach takes into account the age structure of a standard population (in this case, the UN population for developed countries) and the prevalence of dementia for each age group in the study population. The standardized estimate can be seen as the prevalence that would be observed if the study population had the same age structure as the standard population. The prevalence of Alzheimer disease $(\mathrm{AD})$, vascular dementia $(\mathrm{VaD})$ and mixed $\mathrm{AD}$ and $\mathrm{VaD}$ was estimated with respective $95 \% \mathrm{CI}$. The associations between $\mathrm{AD}, \mathrm{VaD}$, and mixed $\mathrm{AD}$ and $\mathrm{VaD}$ with gender were estimated. These analyses were controlled for age group. We estimated the association between dementia and educational attainment as well as income with logistic regression. These analyses were controlled for gender and age group. Statistical significance was assessed with likelihood ratio tests.

\section{Results}

\section{Sample characteristics}

It was necessary to contact (by door-knocking) 21,727 households in 66 census sectors to identify 2,266 persons aged 65 years and over, of whom 2,072 (91.4\%) agreed to participate in the study. Among the 194 non-participants, $180(8.0 \%)$ refused to participate and $14(0.6 \%)$ died or moved to an address outside the study area between the identification and interview phases. Participants and non-participants had similar gender and age distributions. Twenty-five $(1.2 \%)$ participants were severely ill and did not answer any of the cognitive questions. Most informants were the children $(47.0 \%)$ or spouses $(33.6 \%)$ of participants; $76.6 \%$ were women, and the mean age of informants was 50.0 years (range: 16 to 95, median: 49). We were unable to identify an informant for four participants $(0.2 \%)$. We completed the assessment of these subjects and all available information was then used to determine whether or not dementia was present at a consensus meeting of the investigators.

Table 1 shows the demographic and socioeconomic characteristics of the participants. Their ages ranged from 65 to 102 years, $42.9 \%$ were aged 65 to 69 years old, $65.8 \%$ had been born in rural areas, $45.9 \%$ were married, $38.3 \%$ were illiterate and $31.0 \%$ had a monthly income of less than US $\$ 85.00$ (only 29 $(1.4 \%)$ subjects had a monthly income of more than US $\$ 1,000)$. Those living in shanty towns numbered 545 (26.3\%). Of the participants, 1,255 (60.6\%) were 
Table 1. Demographic and socioeconomic characteristics of participants

\begin{tabular}{|c|c|c|c|}
\hline C H A R A C TERIS T I C & $\begin{array}{l}\text { WOMEN } \\
N(\%)\end{array}$ & $\begin{array}{l}M E N \\
N(\%)\end{array}$ & $\begin{array}{l}\text { TOTAL } \\
\mathrm{N}(\%)\end{array}$ \\
\hline \multicolumn{4}{|l|}{ AGE BAND (YEARS) } \\
\hline 65 to 69 & $519(41.3)$ & $370(45.3)$ & $889(42.9)$ \\
\hline 70 to 74 & $339(27.0)$ & $217(26.6)$ & $556(26.8)$ \\
\hline 75 to 79 & $220(17.5)$ & $126(15.4)$ & $346(16.7)$ \\
\hline 80 to 84 & $98(7.8)$ & $71(8.7)$ & $169(8.2)$ \\
\hline 85 or more & $79(6.3)$ & $33(4.0)$ & $112(5.4)$ \\
\hline \multicolumn{4}{|l|}{ PLACE OF BIRTH* } \\
\hline Rural area & $836(66.7)$ & $525(64.5)$ & $1361(65.8)$ \\
\hline Town & $267(21.3)$ & $207(25.4)$ & $474(22.9)$ \\
\hline City & $151(12.0)$ & $82(10.1)$ & $233(11.3)$ \\
\hline \multicolumn{4}{|l|}{ MARITAL STATUS } \\
\hline Married & $339(27.0)$ & $613(75.0)$ & $952(45.9)$ \\
\hline Widowed & $616(49.1)$ & $115(14.1)$ & $731(35.3)$ \\
\hline Never married/separated & $300(23.9)$ & $89(10.9)$ & $389(18.8)$ \\
\hline \multicolumn{4}{|l|}{ EDUCATION (YEARS) } \\
\hline none & $527(42.0)$ & $266(32.5)$ & $793(38.3)$ \\
\hline 1 to 3 & $629(50.1)$ & $454(55.6)$ & $1083(52.3)$ \\
\hline 4 or more & $99(7.9)$ & $97(11.9)$ & $196(9.4)$ \\
\hline \multicolumn{4}{|c|}{ MONTHLY PERSONAL INCOME (US\$) ${ }^{* *}$} \\
\hline None to 85 & $476(37.9)$ & $167(20.4)$ & $643(31.0)$ \\
\hline 86 to 127 & $289(23.0)$ & $113(13.8)$ & $402(19.4)$ \\
\hline 128 to 246 & $285(22.7)$ & $230(28.2)$ & $515(24.9)$ \\
\hline 247 to 500 & $161(12.8)$ & $202(24.8)$ & $363(17.5)$ \\
\hline 501 or more & $44(3.5)$ & $105(12.8)$ & $149(7.2)$ \\
\hline
\end{tabular}

* Four participants with missing values.

** The minimum Brazilian wage at the time of the study was approximately US $\$ 85$.

women, and women were older than men and had lower educational attainment and income.

\section{Prevalence of dementia}

The criteria for the diagnosis of dementia were met in 105 participants, yielding a prevalence of 5.1\% (95\% CI 4.1-6.0) (Table 2) and a standardized population prevalence of $6.6 \%(95 \%$ CI 5.4-7.8). Among women, the prevalence of dementia was $5.4 \%$ (95\% CI 4.2-6.7) and among men $4.5 \%$ (95\% CI 3.1-6.0). The prevalence of dementia increased with age, from $2.3 \%$ among those aged $65-69$ to $16.7 \%$ among those aged 80 years and over.

Of the participants with dementia, 34 (32.4\%) had $\mathrm{AD}, 34(32.4 \%) \mathrm{VaD}$ and $37(35.2 \%)$ other types of dementia (29 cases of mixed $\mathrm{AD}$ and $\mathrm{VaD}$, and eight cases with a history of parkinsonism). The prevalence of $\mathrm{AD}$ was $1.6 \%$ 
Table 2. Prevalence of dementia by gender and age group, and odds ratios (OR) with $95 \%$ confidence intervals for the association of dementia with age group by gender

\begin{tabular}{|c|c|c|c|c|c|}
\hline $\begin{array}{l}\text { A GE } \\
\text { GROUP } \\
\text { (YEARS) }\end{array}$ & $\mathrm{N}(\%)$ & $\begin{array}{l}\text { N UMBER } \\
\text { WITH } \\
\text { DEMENTIA }\end{array}$ & $\begin{array}{l}\text { P R E VA LEN C E } \\
\left(\begin{array}{lll}95 \% & \text { C I })\end{array}\right.\end{array}$ & $\begin{array}{l}\text { O D D S } \\
\left(\begin{array}{ll}95 \% & \text { C I I })\end{array}\right.\end{array}$ & $P-V A L U E^{1}$ \\
\hline \multicolumn{6}{|l|}{ FEMALE } \\
\hline $65-69$ & $519(41.4)$ & 11 & $2.1(0.9-3.4)$ & 1 & $<0.001$ \\
\hline $70-74$ & $339(27.0)$ & 6 & $1.8(0.4-3.2)$ & $0.83(0.30-2.27)$ & \\
\hline $75-79$ & $220(17.5)$ & 17 & $7.7(4.2-11.3)$ & $3.87(1.78-8.40)$ & \\
\hline 85 or more & $79(6.3)$ & 19 & $24.1(14.4-33.7)$ & $14.62(6.64-32.20)$ & \\
\hline \multicolumn{6}{|l|}{ MALE } \\
\hline $65-69$ & $370(45.3)$ & 9 & $2.4(0.9-4.0)$ & 1 & $<0.001$ \\
\hline $70-74$ & $217(26.6)$ & 5 & $2.3(0.3-4.3)$ & $0.95(0.31-2.86)$ & \\
\hline $75-79$ & $126(15.4)$ & 10 & $7.9(3.2-12.7)$ & $3.46(1.37-8.72)$ & \\
\hline $80-84$ & $71(8.7)$ & 8 & $11.3(3.7-18.8)$ & $5.09(1.89-13.70)$ & \\
\hline 85 or more & $33(4.0)$ & 5 & $15.2(2.2-28.1)$ & $7.16(2.25-22.82)$ & \\
\hline $65-69$ & 889 (42.9) & 20 & $2.3(1.3-3.2)$ & 1 & $<0.001$ \\
\hline $70-74$ & $556(26.8)$ & 11 & $2.0(0.8-3.1)$ & $0.88(0.42-1.84)$ & \\
\hline $75-79$ & $346(16.7)$ & 27 & $7.8(5.0-10.6)$ & $3.68(2.03-6.65)$ & \\
\hline $80-84$ & $169(8.2)$ & 23 & $13.6(8.4-18.8)$ & $6.84(3.66-12.78)$ & \\
\hline 85 or more & $112(5.4)$ & 24 & $21.4(13.7-29.1)$ & $11.85(6.29-22.31)$ & \\
\hline
\end{tabular}

${ }^{1} \mathrm{p}$-value for trend 
(95\% CI 1.1-2.2), with a non-significant trend for higher prevalence among women $(\mathrm{OR}=1.94,95 \%$ CI $0.87-4.35$; adjusted for age). The prevalence of $\mathrm{VaD}$ was $1.6 \%(95 \%$ CI $1.2-2.2)$ and was higher among men than women (OR $=2.17,95 \%$ CI 1.09-4.33; adjusted for age). Prevalence of mixed dementia was $1.4 \%(95 \%$ CI $0.9-1.9)$ and affected a similar proportion of men and women $(\mathrm{OR}=0.75,95 \% \mathrm{CI}$ 0.34-1.66; adjusted for age).

Low educational attainment and income were associated with increased risk of dementia, even after the analyses were controlled for gender and age group. Using no formal education as the reference category, the OR for those with one to three years of formal education and for those with four or more years were 0.75 and 0.19 , respectively $(\mathrm{p}<0.001$, test for trend). When considering the lower income group as the reference, the OR for the income groups between US $\$ 86$ and US $\$ 127$, US $\$ 128$ and US $\$ 246$, and over US $\$ 247$ were $0.60,0.34$, and 0.27 , respectively $(\mathrm{p}<0.001$, test for linear trend). Education and income did not change the association between age group and dementia. Gender was not associated with dementia even after taking education and income into account.

\section{Discussion}

The results of this study showed that the prevalence of dementia among disadvantaged people aged 65 years or over is approximately $5 \%$, with similar prevalence for men and women in all age groups up to age 80 years, after which a larger proportion of women are affected. Low educational attainment and income were associated with increased risk of dementia. The implication of these findings will be discussed after consideration of the methodological aspects of our survey.

\section{Methodological aspects}

Non-participation was low (8.6\%) and mostly due to refusals. The one-stage design avoided systematic losses between identification of eligible subjects and the assessment of cognitive status. Institutionalized older people were not included, but this is unlikely to have altered the prevalence estimate, as people from low socioeconomic status in São Paulo do not have access to residential services (nursing homes or hostels), which are run entirely by the private sector. Because the study aimed to assess the prevalence of dementia in a disadvantaged community, its results may fail to reflect accurately the situation for the entire population of São Paulo or Brazil. However, our results provide valuable information about the situation for a large proportion of older Brazilians.

Differences in prevalence estimates of dementia between studies may be explained by methodological differences. For example, the authors of a study carried out in four centres of China (Zhang et al., 2005) attributed the higher prevalence of dementia in their study to ascertainment bias and small sample sizes of previous studies in East Asia. In the present study, the diagnosis of dementia was based on the use of a well-established protocol with robust 
sensitivity and specificity (Scazufca et al., 2002; Prince et al., 2003). This protocol asks all informants about the cognitive and functional status of participants regardless of their cognitive status, which improves the quality of the information obtained (Prince et al., 2003). We acknowledge that the classification of dementia subtypes could have been improved with the use of a more accurate clinical neurological evaluation and neuroimaging. However, it is now widely accepted that $\mathrm{AD}$ and $\mathrm{VaD}$ show significant overlap and that misclassification of these dementia subtypes might have limited clinical implications (Langa et al., 2004). Inaccuracies in the ascertainment of age could have potentially biased the estimate of age-specific prevalence, although this was not a common occurrence in this study (uncertainty in relation to participants' ages occurred in only 39 subjects, and for 25 subjects it was limited to a maximum of two years).

\section{Prevalence of dementia}

The prevalence of dementia in our study is in the lower range of what has been reported by surveys conducted in developed economies (Hendrie et al., 1995; Breitner et al., 1999; Lobo et al., 2000), but is similar to findings from four centers in China (Zhang et al., 2005), and higher than in India, Nigeria and Sri Lanka (Hendrie et al., 1995; Vas et al., 2001; de Silva et al., 2003; Shaji et al., 2005). Our study sample had a relatively large proportion of people aged 65 to 69 years, and this may partly explain the relatively low prevalence of dementia when compared to developed countries. In fact, the standardized prevalence estimate that uses the United Nations World Population Prospect for developed countries (United Nations Department of Economic and Social Affairs, 2005) confirmed that our results are largely in agreement with those of Europe, North America and Australia.

We did not observe the typical exponential rise in the prevalence of dementia with increasing age reported by previous studies (Hendrie et al., 1995; Lobo et al., 2000). Instead, the prevalence of dementia in the age groups 65-69 and 70-74 years was all but identical. This finding might be due to the high prevalence of cerebrovascular disease and other comorbid conditions in this population (Lotufo, 2005), failure to compress morbidity into the very latter stages of life (aged $80+$ ), high mortality among those with dementia (early censoring of cases), and limited access to health care (Lima-Costa et al., 2003; Dufour and Piperata, 2004). However, lack of precision for age-specific prevalence estimates does not allow a firmer conclusion about these possibilities. Our findings are also consistent with those recently reported in a comprehensive review of dementia in the United Kingdom (Alzheimer's Society, 2007). The report confirmed that the prevalence of $\mathrm{AD}$ is higher among women (67\% versus $55 \%$ ), whereas $\mathrm{VaD}$ and mixed cases are more prevalent in men (31\% versus $25 \%$ ). Similar findings have been reported by others (for example, Alzheimer's Australia, 2003).

Numerous other studies support our finding that low educational attainment is associated with increased risk of dementia, as is low income (reviewed by Valenzuela and Sachdev, 2006). It is interesting that the association between dementia and poor education and low income was observed in this particularly deprived community, which has a substantially narrower range of education 
and income than the samples recruited for the studies in developed countries. This suggests that even small gains in those factors could potentially lead to a significant reduction in the prevalence of dementia in the world.

With the progressive improvement of medical care in developing countries, more older adults with dementia will reach their 70s and 80 s, resulting in a significant increase in the total number of people with dementia. If that is the case, the number of people with dementia living in developing countries by 2040 may be even higher than the expected 70\% (Ferri et al., 2005). In Brazil, as in many developing countries, there are only limited social policies and scarce resources to support health programs for older people (Lloyd-Sherlock, 2000), making family members, usually women, the most important source of care for people who develop dementia (10/66 Dementia Research Group, 2004). In large urban centers, family size is rapidly decreasing, and engagement in employment outside the home is needed, making provision of care by family members more difficult. Health care policies and programs accessible to disadvantaged older adults must be implemented as a matter of urgency to cater for the needs of this growing segment of the population of developing countries.

\section{Conflict of interest}

None.

\section{Description of authors' roles}

M. Scazufca and P. R. Menezes designed the study, supervised data collection, planned and carried out the statistical analyses, and drafted the paper. H. P. Vallada contributed to designing the study and drafting the paper. A. L. Crepaldi, V. D. Di Rienzo assisted with data collection and provided critical comments to an earlier draft of this paper. L. M. S. Coutinho contributed to data analysis and helped draft the manuscript. M. Pastor-Valero and O. P. Almeida contributed to the analysis of the data, interpretation of the results and drafting of the manuscript.

\section{Acknowledgments}

The study was funded by the Wellcome Trust, U.K. (grant code GR066133MA). M. Scazufca was supported by the Welcome Trust and CNPq-Brazil during the development of this work. P. R. Menezes and H. P. Vallada were partly funded by CNPQ-Brazil. We thank Professors Martin Prince, John R. M. Copeland and Paul McKeigue for helping with the acquisition of funding. We thank the research team that contributed to the data collection, Professor Paulo Lotufo and the staff of the University Hospital of the University of São Paulo for accommodating this project throughout the study period, all colleagues from the 10/66 Dementia Research Group who helped with the development of the protocol used in São Paulo, as well as the participants of the study. 


\section{References}

10/66 Dementia Research Group (2000a). Methodological issues for population-based research into dementia in developing countries: a position paper from the 10/66 Dementia Research Group. International fournal of Geriatric Psychiatry, 15, 21-30.

10/66 Dementia Research Group (2000b). Dementia in developing countries: a consensus statement from the 10/66 Dementia Research Group. International fournal of Geriatric Psychiatry, 15, 14-20.

10/66 Dementia Research Group (2004). Care arrangements for people with dementia in developing countries. International fournal of Geriatric Psychiatry, 19, 170-177.

Alzheimer's Australia (2003). The Dementia Epidemic: Economic Impact and Positive Solutions for Australia. http://www.alzheimers.org.au/upload/EpidemicFullReportMarch2003.pdf; accessed 20 March 2007.

Alzheimer's Society (2007). Dementia UK: The Full Report. http://www.alzheimers.org.uk/News_ and_Campaigns/Campaigning/PDF/Dementia_UK_Full_Report.pdf; accessed 20 March 2007.

American Psychiatric Association (1994). Diagnostic and Statistic Manual of Mental Disorders: $D S M-I V$, 4th edn. Washington, DC: American Psychiatric Association.

Breitner, J. C. et al. (1999). APOE- $\epsilon 4$ count predicts age when prevalence of AD increases, then declines: the Cache County Study. Neurology, 53, 321-331.

Cohen, J. E. (2003). Human population: the next half century. Science, 302, 1172-1175.

Copeland, J. R., Dewey, M. E. and Griffiths-Jones, H. M. (1986). A computerized psychiatric diagnostic system and case nomenclature for elderly subjects: GMS and AGECAT. Psychological Medicine, 16, 89-99.

de Silva, H. A., Gunatilake, S. B. and Smith, A. D. (2003). Prevalence of dementia in a semi-urban population in Sri Lanka: report from a regional survey. International fournal of Geriatric Psychiatry, 18, 711-715.

Dewey, M. E. and Copeland, J. R. (2001). Diagnosis of dementia from the history and aetiology schedule. International fournal of Geriatric Psychiatry, 16, 912-917.

Dufour, D. L. and Piperata, B. A. (2004). Rural-to-urban migration in Latin America: an update and thoughts on the model. American fournal of Human Biology, 16, 395-404.

Ferri, C. P. et al. (2005). Global prevalence of dementia: a Delphi consensus study. Lancet, 366, 2112-2117.

Fries, J. F. (1980). Aging, natural death, and the compression of morbidity. New England fournal of Medicine, 303, 130-135.

Hall, K. S., Gao, S., Emsley, C. L., Ogunniyi, A. O., Morgan, O. and Hendrie, H. C. (2000). Community screening interview for dementia (CSI ' $D$ '); performance in five disparate study sites. International fournal of Geriatric Psychiatry, 15, 521-531.

Hendrie, H. C. et al. (1995). Prevalence of Alzheimer's disease and dementia in two communities: Nigerian Africans and African Americans. American fournal of Psychiatry, 152, 1485-1492.

Herrera, E. Jr., Caramelli, P., Silveira, A. S. and Nitrini, R. (2002). Epidemiologic survey of dementia in a community-dwelling Brazilian population. Alzheimer Disease and Associated Disorders, 16, 103-108.

Hofman, A. et al. (1991). The prevalence of dementia in Europe: a collaborative study of 1980-1990 findings. EURODEM Prevalence Research Group. International Fournal of Epidemiology, 20, 736-748.

Instituto Brasileiro de Geografia e Estatística (2006). Censo 2000: Brazil. http://www.ibge.gov.br/censo/; accessed 20 March 2007

Jorm, A. F., Korten, A. E. and Henderson, A. S. (1987). The prevalence of dementia: a quantitative integration of the literature. Acta Psychiatrica Scandinavica, 76, 465-479. 
Langa, K. M., Foster, N. L. and Larson, E. B. (2004). Mixed dementia: emerging concepts and therapeutic implications. $\mathcal{F} A M A, 292,2901-2908$.

Lima-Costa, M. F., Barreto, S., Giatti, L. and Uchoa, E. (2003). [Socioeconomic circumstances and health among the Brazilian elderly: a study using data from a National Household Survey]. Cadernos de Saude Publica, 19, 745-757.

Lloyd-Sherlock, P. (2000). Population ageing in developed and developing regions: implications for health policy. Social Science and Medicine, 51, 887-895.

Lobo, A. et al. (2000). Prevalence of dementia and major subtypes in Europe: a collaborative study of population-based cohorts. Neurologic Diseases in the Elderly Research Group. Neurology, 54, S4-S9.

Lotufo, P. A. (2005). Stroke in Brazil: a neglected disease. São Paulo Medical fournal, 123, 3-4.

Marins, V. M., Almeida, R. M., Pereira, R. A. and Sichieri, R. (2007). The association between socioeconomic indicators and cardiovascular disease risk factors in Rio de Janeiro, Brazil. Fournal of Biosocial Science, 39, 221-229.

Newman, A. B. et al. (2005). Dementia and Alzheimer's disease incidence in relationship to cardiovascular disease in the Cardiovascular Health Study cohort. Fournal of the American Geriatrics Society, 53, 1101-1107.

Prince, M., Acosta, D., Chiu, H., Scazufca, M. and Varghese, M. (2003). Dementia diagnosis in developing countries: a cross-cultural validation study. Lancet, 361, 909-917.

Prince, M. et al. (2004). Effects of education and culture on the validity of the Geriatric Mental State and its AGECAT algorithm. British Fournal of Psychiatry, 185, 429-436.

Scazufca, M. et al. (2002). [Epidemiological research on dementia in developing countries]. Revista de Saude Publica, 36, 773-778.

Secretaria do Governo da Prefeitura de São Paulo (2004). Sumário de Dados do Município de São Paulo 2004. http://portal.prefeitura.sp.gov.br/secretarias/governo/sumario_dados; accessed 20 March 2007.

Shaji, S., Bose, S. and Verghese, A. (2005). Prevalence of dementia in an urban population in Kerala, India. British fournal of Psychiatry, 186, 136-140.

StataCorp (2005). Stata Statistical Software, Release 9. College Station, TX: StataCorp.

United Nations Department of Economic and Social Affairs (2002). World Population Ageing 1950-2050. http://www.un.org/esa/population/publications/worldageing19502050/; accessed 20 March 2007.

United Nations Department of Economic and Social Affairs (2005). World Population Prospects: The 2004 Revision Population Dataset. New York: United Nations.

Valenzuela, M. J. and Sachdev, P. (2006). Brain reserve and dementia: a systematic review. Psychological Medicine, 36, 441-454.

Vas, C. J. et al. (2001). Prevalence of dementia in an urban Indian population. International Psychogeriatrics, 13, 439-450.

Welsh, K. A. et al. (1994). The Consortium to Establish a Registry for Alzheimer's Disease (CERAD). Part V. A normative study of the neuropsychological battery. Neurology, 44, 609-614.

Zhang, Z. X. et al. (2005). Dementia subtypes in China: prevalence in Beijing, Xian, Shanghai, and Chengdu. Archives of Neurology, 62, 447-453. 